\title{
ORIGINAL RESEARCH \\ Measurement of Gray and White Matter Atrophy in Dementia with Lewy Bodies Using Diffeomorphic Anatomic Registration through Exponentiated Lie Algebra: A Comparison with Conventional Voxel-Based Morphometry
}

R. Takahashi

K. Ishii

N. Miyamoto

T. Yoshikawa

K. Shimada

S. Ohkawa

T. Kakigi

K. Yokoyama

\begin{abstract}
BACKGROUND AND PURPOSE: DLB is recognized as the second major form of dementia in the elderly. The regional pattern of GM atrophy in DLB highly overlaps that in AD. The aim of this study was to identify the critical pattern of atrophy in DLB by using DARTEL, which provides improved registration accuracy compared with that of conventional VBM.
\end{abstract}

MATERIALS AND METHODS: We evaluated 51 patients with probable AD, 43 patients with probable DLB, and 40 age-matched healthy controls. The pattern of GM atrophy in each group was compared by using conventional VBM and VBM-DARTEL.

RESULTS: Regional patterns of atrophy identified by using conventional VBM differed significantly from those identified by using VBM-DARTEL. A decrease in GM volume in the MTLs in both AD and DLB was identified with VBM-DARTEL; the decrease was greater in patients with $A D$ than in those with DLB. Comparisons with healthy controls revealed that the WM volume of the whole brain was preserved in patients with DLB. In contrast, a severe bilateral decrease in WM in the MTLs was detected in patients with $A D$.

CONCLUSIONS: VBM-DARTEL provided more accurate results, and it enabled the identification of more localized morphologic alterations than did conventional VBM. Analysis of WM preservation in DLB could help to differentiate this condition from AD.

\begin{abstract}
ABBREVIATIONS: $A D=$ Alzheimer disease; $\mathrm{DARTEL}=$ Diffeomorphic Anatomical Registration Through Exponentiated Lie algebra; DLB = dementia with Lewy bodies; GM = gray matter; Lt = left; MMSE = Mini-Mental State Examination; MTL = medial temporal lobe; Rt = right; SE = spin-echo; SPGR = spoiled gradient-recalled; VBM = voxel-based morphometry; WM = white matter
\end{abstract}

D $\mathrm{LB}$ is recognized as the second major form of dementia in the elderly. The main symptoms of DLB are visual hallucinations, fluctuating cognitive impairment, and parkinsonism. ${ }^{1}$ DLB is clinically and neuropathologically different from AD. However, a definitive diagnosis can only be made on the basis of postmortem neuropathologic findings. Recent in vivo imaging studies using fluorodeoxyglucosepositron-emission tomography ${ }^{2-5}$ and single-photon emission $\mathrm{CT}^{6,7}$ aided in the establishment of criteria for DLB diagnosis. These studies revealed that DLB is characterized by low glucose metabolism and decreased regional cerebral blood flow in the occipital cortex, which distinguishes it from AD. ${ }^{8-10}$

On the other hand, structural changes in DLB were found to closely resemble those in $\mathrm{AD}$. Volumetric studies have con-

\section{Received March 5, 2010; accepted after revision April 27}

From the Departments of Neurology (R.T., K.Y.) and Cognitive Disorders (T.K.), Hyogo Prefectural Rehabilitation Hospital at Nishi-Harima, Tatsuno, Hyogo, Japan; Department of Radiology and Nuclear Medicine (K.I., N.M., T.Y.) and Institute for Aging Brain and Cognitive Disorders (K.S., S.O.), Hyogo Brain and Heart Center, Himeji, Hyogo, Japan; and Department of Radiology (K.I.), Kinki University School of Medicine, Osakasayama, Osaka, Japan.

Please address correspondence to K. Ishii, MD, Department of Radiology, Kinki University School of Medicine, 377-2 Ohnohigashi, Osakasayama, Osaka 589-8511, Japan; e-mail: kishii@hbhc.jp

DOI 10.3174/ajnr.A2200 sistently shown a loss of GM in the MTLs, including the hippocampus and amygdala, in patients with DLB. ${ }^{11,12}$ Although the regional patterns of cerebral GM atrophy in DLB overlap those of $\mathrm{AD}$, the degree of GM loss in DLB is much lower than that in $\mathrm{AD} .{ }^{11,13} \mathrm{VBM}$ was developed to assess the whole brain structure with a voxel-by-voxel comparison. ${ }^{14-16}$ Researchers have attempted to identify the specific pattern of atrophy in DLB with VBM and have observed similar atrophy with relative preservation of the temporal lobes in DLB and AD. ${ }^{17-19}$

However, studies using different technical approaches have found that the extent of GM reduction in DLB differs between the putamen ${ }^{20}$ and basal forebrain, including the substantia innominata. ${ }^{21}$ Whitwell et al ${ }^{19}$ conducted a VBM study with a relatively large number of subjects and found no DLB-specific differences by voxel-by-voxel GM comparison; however, by using VBM-based region-of-interest analysis, they suggested that the GM attenuation in the dorsal midbrain was lower in DLB than in AD. However, the results of region-of-interest analyses are quite dependent on the arbitrary locus and size that the examiners create retrospectively after the VBM analysis. In addition, Whitwell et al did not address the WM alterations in DLB.

Recently, DARTEL (SPM8; Wellcome Trust Centre for Neuroimaging, London, United Kingdom), a fast diffeomorphic registration algorithm, has been developed for use 


\begin{tabular}{lccc}
\hline \multicolumn{4}{l}{ Table 1: Characteristics of the subjects } \\
\hline & Patients with DLB & Patients with AD & Controls \\
\hline Median age (yr) & $72.7 \pm 4.5$ & $72.6 \pm 2.9$ & $72.0 \pm 3.8$ \\
Median MMSE score & $19.0 \pm 3.5$ & $18.7 \pm 4.0$ & $29.6 \pm 0.8$ \\
Number of women (\%) & $60 \%$ & $61 \%$ & $50 \%$ \\
Neuropsychological & Visual hallucination: & \\
examinations (\%) & $61 \%$ \\
& Cognitive fluctuation: \\
& $95 \%$ \\
& Parkinsonism: 84\% \\
\hline
\end{tabular}

with VBM. ${ }^{22}$ This involves creating a mean image of all those taken, which serves as a subject-specific template. Subsequently, whole-brain images of individual subjects are normalized to the template, modulated, and smoothed. DARTEL was shown to improve registration and provide precise and accurate localization of structural damage and functional overlays. The aim of the present study was to investigate more precise voxel-based morphometric comparisons in GM and WM reduction between DLB and AD. Our hypothesis was that the specific patterns of GM and WM loss in DLB would be more clearly demonstrated with DARTEL than with conventional VBM.

\section{Materials and Methods}

In this study, we included 43 patients with probable DLB, 51 patients with probable $\mathrm{AD}$, and 40 cognitively healthy controls. We retrospectively selected subjects from among patients who were admitted to the infirmary at our institution for an evaluation of dementia between April 2002 and March 2006, and the patients were followed up for $>3$ years. The subjects were matched for sex and age, and their MMSE score was calculated. All the patients were examined by neurologists and psychiatrists and underwent standard neurologic and neuropsychological examinations, laboratory tests, electroencephalography, and cranial MR imaging. Clinical data revealed fluctuations in cognitive functions, recurrent visual hallucinations, and spontaneous parkinsonism; these data were used to diagnose probable DLB on the basis of the criteria proposed in the Consortium on DLB International Workshop. $^{23}$

Patients with DLB showed symptoms of visual hallucination (61\%), cognitive fluctuation (95\%), and parkinsonism (84\%). Patients with $\mathrm{AD}$ fulfilled the criteria for probable $\mathrm{AD}$ outlined by the National Institute of Neurologic and Communicative Disorders and Stroke/Alzheimer Disease and Related Disorders Association. None of the patients with AD exhibited parkinsonism. All the control subjects had an MMSE score of $>28$ and had no clinical evidence of cognitive deficits or neurologic diseases. None of the subjects exhibited abnormal MR imaging findings, except for age-related brain atrophy and WM hyperintensity. The clinical characteristics of each group (mean age, mean MMSE score, percentage of women, and the results of neuropsychological examinations) are shown in Table 1.

\section{MR Imaging Procedures}

A 1.5T Signa Horizon MR imaging system was used for this study (GE Healthcare, Milwaukee, Wisconsin). Sagittal, coronal, and axial T1weighted SE images (550/15 ms/2 [TR/TE/NEX], 5-mm thickness, 2.5-mm gap) and axial T2-weighted fast SE images (3000/105 ms/2 [TR/TE/NEX]) were obtained to detect abnormalities. VBM analyses were conducted by using coronal 3D SPGR images (14/3 ms/
2 [TR/TE/NEX], $20^{\circ}$ flip angle, $220-\mathrm{mm}$ FOV, $256 \times 256$ matrix $124 \times 1.5 \mathrm{~mm}$ contiguous sections).

\section{Data Analysis}

Statistical analyses were conducted by using the Statistical Parametric Mapping, Version 8 software for Windows (SPM8). Calculations and image matrix manipulations were performed by using MATLAB (MathWorks, Natick, Massachusetts). For VBM analysis by using SPM 8, all coronal SPGR MR imaging datasets were reconstructed to axial datasets and subsequently converted to the ANALYZE format (http://www.mayo.edu/bir/Software/Analyze/Analyze.html) and displayed with the right hemisphere on the right side of the image. MR imaging data were analyzed by using VBM implemented in SPM8. First, conventional VBM was performed by using the default template. Next, we applied DARTEL, an algorithm for accurate diffeomorphic image registration, implemented as a toolbox for SPM 8, to create a set of group-specific templates. The brain images were segmented, normalized, and modulated by using these templates. The output images were still in the average brain space. Additional warping from the Montreal Neurologic Institute space was given to the brain images. Finally, GM probability values were smoothed by using a 6-mm full width at half maximum Gaussian kernel. The DARTEL procedure improves anatomic precision in addition to the previous spatial normalization methods created by Ashburner and Friston. ${ }^{14,16}$

\section{Group Comparisons of Regional GM and WM Loss}

First, we compared regional GM and WM loss in healthy controls with those in patients with $\mathrm{DLB}$ and those with $\mathrm{AD}$, respectively. Then, the GM and WM losses between DLB and patients with AD were compared. Each group comparison was analyzed by a statistical design with the 1-way analysis of variance test. In this study, age and total intracranial volume were entered as covariates. Significance was accepted if the voxels survived a corrected threshold of $P<.001$ for GM and WM comparisons versus healthy controls and an uncorrected threshold of $P<.001$ in all other comparisons.

\section{Results}

Compared with the healthy controls, both patients with $\mathrm{AD}$ and those with DLB showed greatly decreased GM in the MTLs (Fig 1 and Tables 2 and 3). In comparison with patients with $\mathrm{AD}$, patients with DLB showed a relatively localized bilateral decrease in GM in the temporal lobes (Fig 1 and Tables 2 and 3). This pattern of results was similar, regardless of whether conventional VBM or VBM-DARTEL was used. A comparison between the groups showed that the bilateral decrease in GM volume in the MTLs was significantly higher in the $\mathrm{AD}$ group than in the DLB group (Fig 2 and Tables 2 and 3). VBM-DARTEL revealed no significant GM decrease in patients with DLB compared with patients with AD; however, conventional VBM revealed a scattered decrease in the GM of the deep brain in patients with DLB (Fig 2 and Tables 2 and 3).

Compared with healthy controls, patients with $\mathrm{AD}$ showed a bilateral decrease in the WM in the MTLs, parieto-occipital lobes, and frontal lobes. VBM-DARTEL revealed no significant decrease in WM in patients with DLB compared with healthy controls. In contrast, conventional VBM revealed that the MTLs and deep brain had significantly decreased WM (Fig 3 and Tables 4 and 5). Comparisons between patient groups revealed more widespread atrophy of WM in patients with $\mathrm{AD}$ than in patients with DLB (Fig 4 and Tables 4 and 5). In com- 

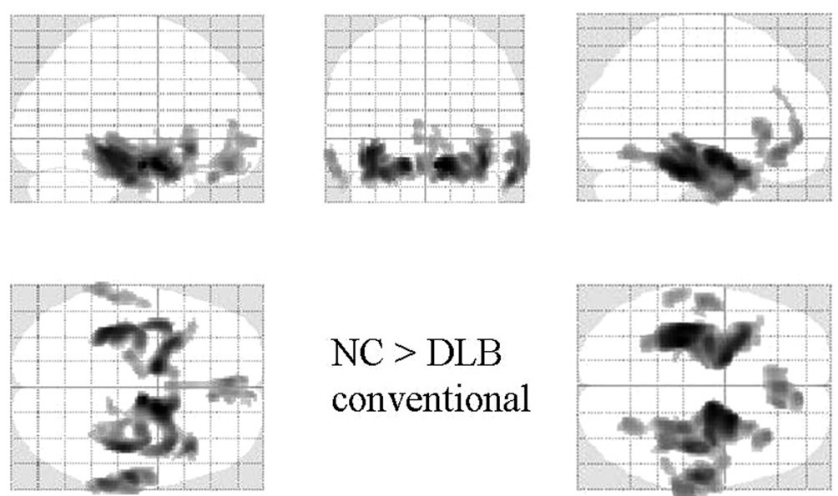

\section{$\mathrm{NC}>\mathrm{DLB}$ conventional}
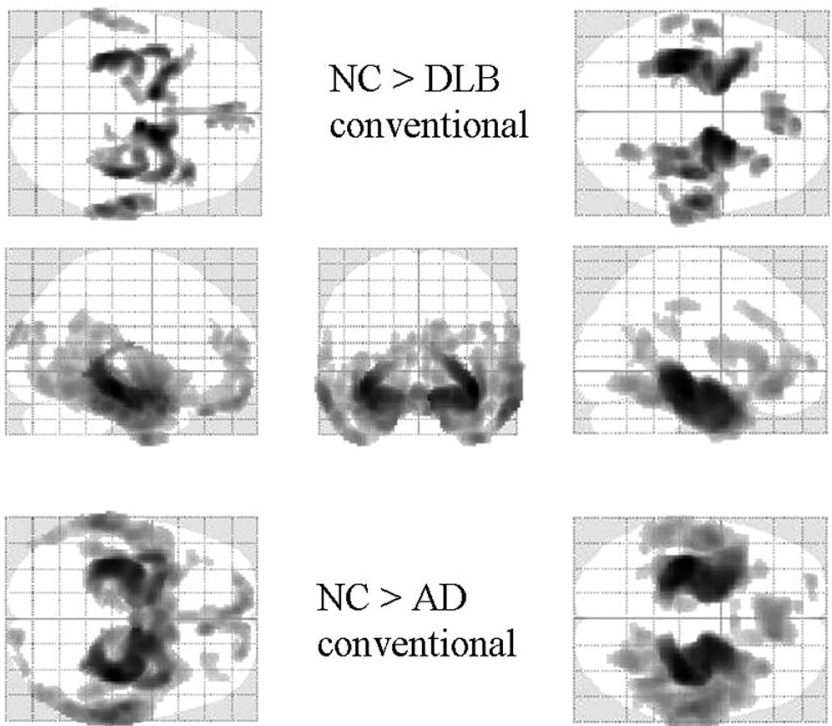

$\mathrm{NC}>\mathrm{AD}$ conventional

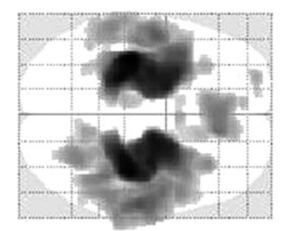

Table 2: Regions in which VBM-DARTEL identified significantly reduced GM: comparisons between patients and healthy controls

\begin{tabular}{|c|c|c|c|c|c|}
\hline \multirow[b]{2}{*}{ Comparison and Brain Region } & \multicolumn{4}{|c|}{ Talairach Coordinates } & \multirow[b]{2}{*}{$t$ Value } \\
\hline & Side & $x$ & Y & Z & \\
\hline \multicolumn{6}{|l|}{$\begin{array}{l}\text { Patients with DLB }<\text { controls } \\
\text {. }\end{array}$} \\
\hline Fusiform gyrus & $\mathrm{Lt}$ & -30 & -30 & -16 & 8.56 \\
\hline Amygdala & Rt & 22 & 2 & -15 & 8.40 \\
\hline Medial frontal lobe & & 0 & 37 & -9 & 6.90 \\
\hline \multicolumn{6}{|l|}{ Patients with $\mathrm{AD}<$ controls } \\
\hline Medial temporal lobe & $\mathrm{Lt}$ & -36 & -24 & -10 & 12.13 \\
\hline Medial temporal lobe & Rt & 37 & -25 & -14 & 11.82 \\
\hline Anterior cingulate gyrus & Rt & 10 & 33 & -13 & 8.08 \\
\hline \multicolumn{6}{|c|}{ Patients with DLB > patients with $A D$} \\
\hline Parahippocampal gyrus & $\mathrm{Lt}$ & -23 & -36 & -2 & 6.62 \\
\hline Medial temporal lobe & Rt & 28 & -29 & -4 & 5.84 \\
\hline
\end{tabular}

parison with patients with $\mathrm{AD}$, patients with DLB showed no WM decrease in both conventional VBM and VBM-DARTEL algorithms.

\section{Discussion}

In the present study, we used the VBM-DARTEL algorithm and found no significant GM reduction in patients with DLB compared with patients with $\mathrm{AD}$. In contrast, conventional VBM detected scattered deep-brain GM reductions in patients with DLB compared with patients with AD (Fig 2). This difference in results is primarily due to the different analytic approach. Unlike the conventional VBM, registration of images is highly complicated in DARTEL. The procedure begins with creating rigidly transformed versions of the tissue class images; in the conventional VBM, this is called "modulation." The next step is creating a mean of all the images to produce an initial template. Deformations from this template to each of the individual images are computed, and the template is then

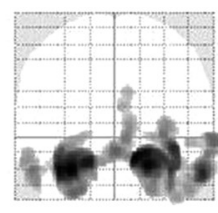

Fig 1. Statistical parametric maps comparing the GM volume of patients with that of age-matched healthy controls (NC). Comparisons based on conventional VBM (left) and VBMDARTEL (right) are both illustrated. Highlighted areas represent regions in which patients have significantly decreased GM compared with controls $(P<.001$, corrected). The regions in which patients with $D L B$ and those with $A D$ show reductions in GM compared with controls overlapped: They were the medial temporal and frontal lobes and the middle temporal gyri on both sides. The pattern of GM decrease in $\mathrm{NC}>\mathrm{DLB}$ patients with $A D$ revealed by conventional VBM is more DARTEL scattered than that identified with VBM-DARTEL.

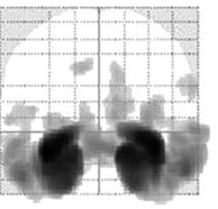

$\mathrm{NC}>\mathrm{AD}$

DARTEL

\begin{tabular}{|c|c|c|c|c|c|}
\hline \multirow[b]{2}{*}{ Comparison and Brain Region } & \multicolumn{4}{|c|}{ Talairach Coordinates } & \multirow[b]{2}{*}{$t$ Value } \\
\hline & Side & $\mathrm{X}$ & Y & Z & \\
\hline \multicolumn{6}{|l|}{ Patients with DLB $<$ controls } \\
\hline Amygdala & Rt & 14 & 0 & -15 & 9.14 \\
\hline Amygdala & Rt & -14 & 0 & 15 & 7.84 \\
\hline Inferior temporal gyrus & Rt & 60 & -19 & -21 & 8.07 \\
\hline \multicolumn{6}{|l|}{ Patients with $\mathrm{AD}<$ controls } \\
\hline Medial temporal lobe & Rt & 38 & -26 & -9 & 12.76 \\
\hline Medial temporal lobe & Lt & -35 & -26 & -9 & 11.85 \\
\hline Superior temporal gyrus & $\mathrm{Lt}$ & -61 & -50 & 14 & 6.90 \\
\hline \multicolumn{6}{|c|}{ Patients with $\mathrm{DLB}>$ patients with $\mathrm{AD}$} \\
\hline Medial temporal lobe & Rt & 34 & -26 & -9 & 5.96 \\
\hline Hippocampus & $\mathrm{Lt}$ & -28 & -11 & -18 & 5.14 \\
\hline
\end{tabular}

regenerated by applying the inverse of the deformations to the images. This is followed by averaging. This procedure is repeated a number of times to create warped versions of the images. The DARTEL toolbox is a high-dimensional warping process that increases the registration between individuals and thereby improves localization and increases sensitivity during analyses.

Thus, the differences in deep brain GM findings may be attributed to the failure of incomplete registration to the default template when using the conventional VBM algorithm. This explanation may also apply to the previous VBM studies in which a significant GM reduction was found in the putamen, ${ }^{20}$ striatum, ${ }^{9}$ and basal forebrain. ${ }^{21}$

Similar to the present study, a previous conventional VBM study using voxel-by-voxel analysis with a large number of subjects revealed that GM decreases did not differ significantly between patients with DLB and those with $\mathrm{AD} .{ }^{19}$ However, the 

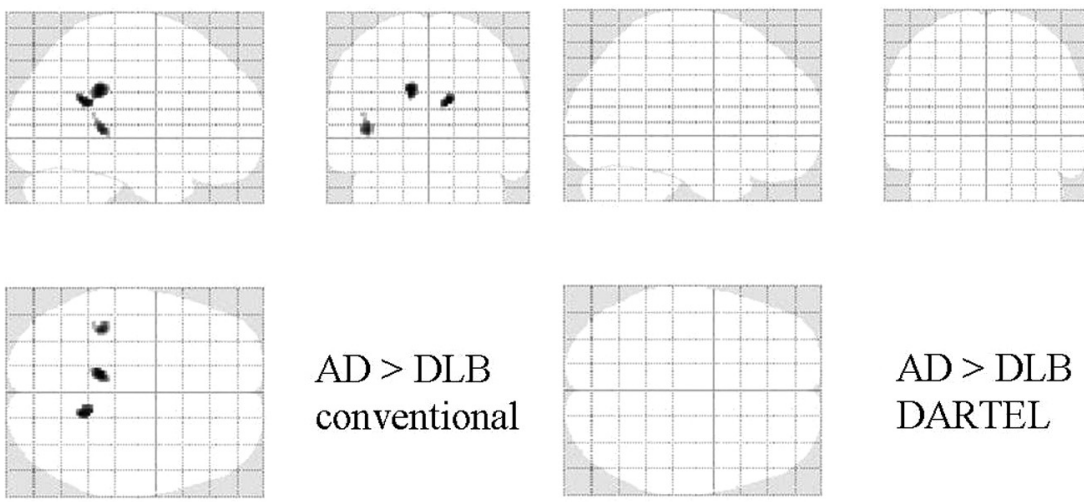

\section{$\mathrm{AD}>\mathrm{DLB}$ conventional}

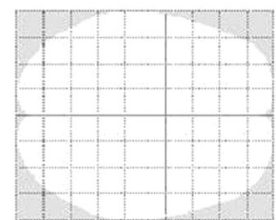

$\mathrm{AD}>\mathrm{DLB}$ DARTEL
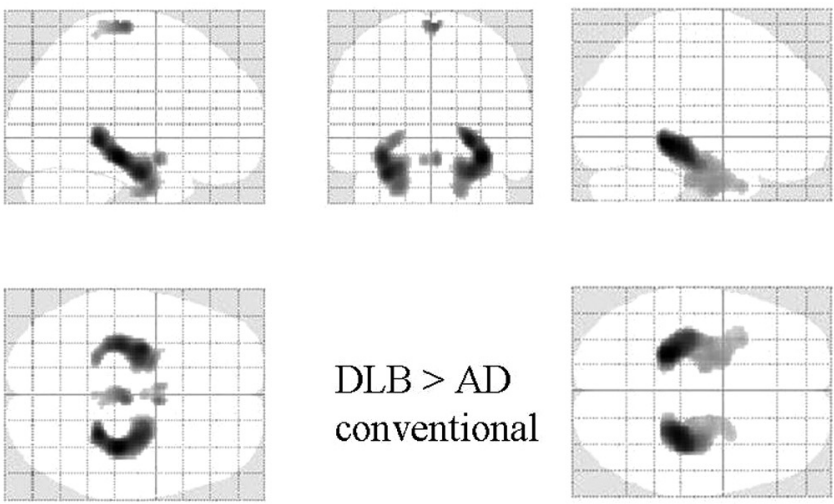
conventional
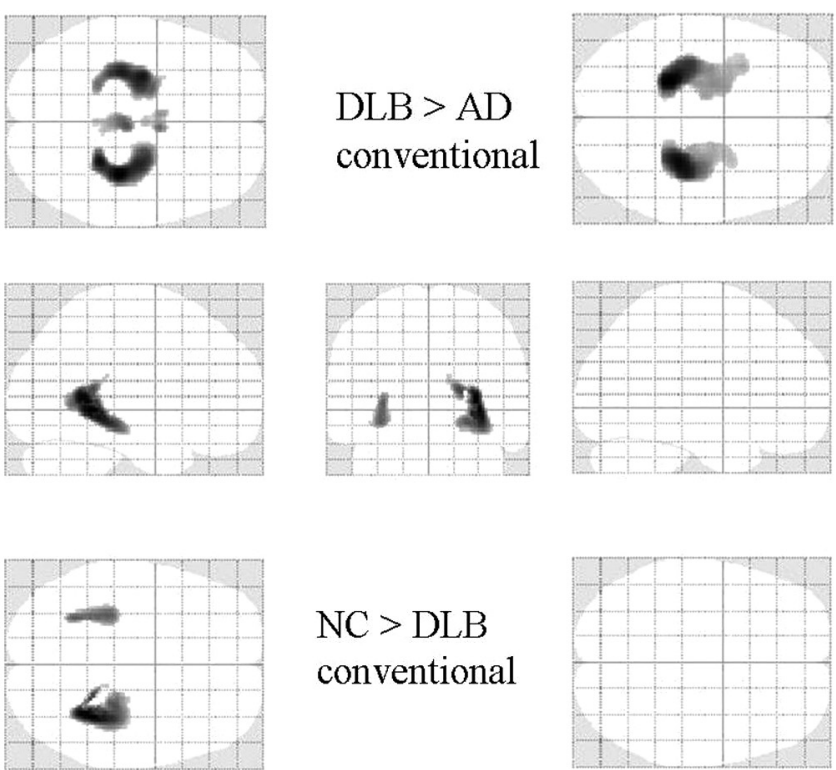

$$
\begin{aligned}
& \mathrm{NC}>\mathrm{DLB} \\
& \text { conventional }
\end{aligned}
$$
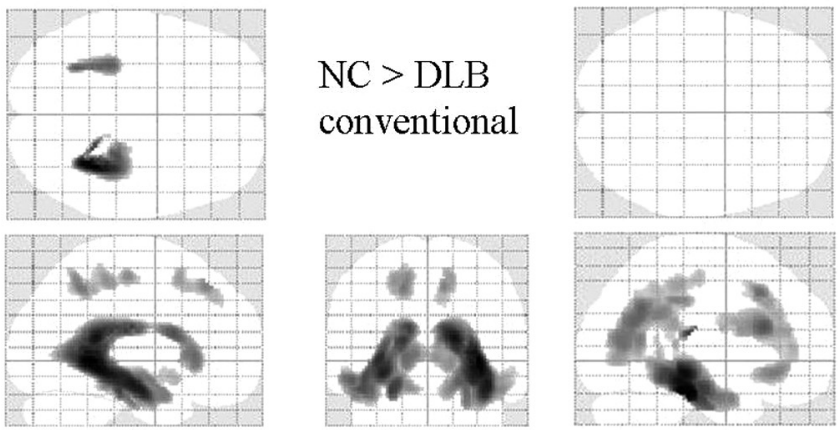

\section{$\mathrm{NC}>\mathrm{DLB}$ DARTEL}
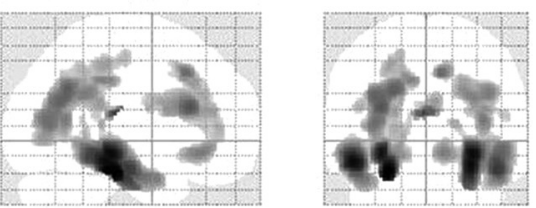

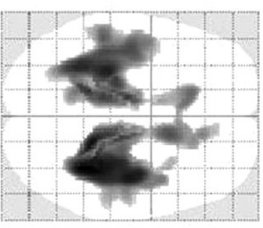

Fig 2. Statistical parametric maps comparing the brains of patients with DLB with those with $A D$. Comparisons based on conventional VBM (left) and VBM-DARTEL (right) are both illustrated. Highlighted areas represent regions in which patients have significantly decreased GM compared with age-matched healthy controls $(P<.001$, uncorrected). $\mathrm{Pa}$ tients with $\mathrm{AD}$ show significant bilateral GM loss in the MTLs. For patients with DLB, the regions in which significant decreases are identified differed between conventional VBM (upper left) and VBM-DARTEL (upper right). While GM decreases are not found in VBM-DARTEL-based comparisons, scattered decreases in the deep brain are identified with conventional VBM.

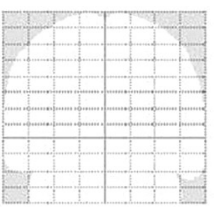

\section{$\mathrm{DLB}>\mathrm{AD}$ \\ DARTEL}

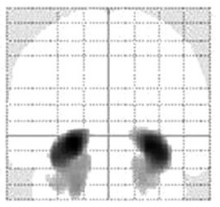

Fig 3. Statistical parametric maps comparing the WM volume of patients with that of age-matched healthy controls (NC). Comparisons based on conventional VBM (left) and VBM-DARTEL (right) are both illustrated. Highlighted areas represent regions in which patients have significantly decreased WM compared with the controls $(P<.001$, uncorrected). Patients with $A D$ show significant WM loss in the bilateral medial temporal, parieto-occipital, and frontal lobes. For patients with DLB, the regions in which significant decreases were identified differ between conventional VBM (upper left) and VBM-DARTEL (upper right). While WM decreases are not found in VBM-DARTEL-based comparisons, decreases in CSF, like WM, are identified in the deep brain with conventional VBM. authors of that study concluded that a GM reduction in the dorsal midbrain found by using region-of-interest analysis with VBM could specifically distinguish patients with DLB from healthy controls. In contrast, there is no pattern of atrophy that differentiates patients with DLB and those with AD. Regions of the brain stem, such as the midbrain, are extremely small, and precise and accurate registration of images is re-

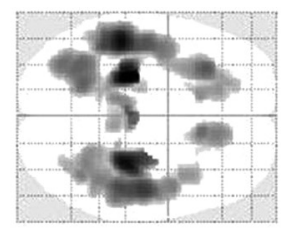

$\mathrm{NC}>\mathrm{AD}$

DARTEL

quired to detect subtle differences between the DLB and AD patient groups. Thus, precise and accurate registration is essential before performing region-of-interest analysis with VBM on modulated GM images, to obtain reliable results. In the present study, even with the highly accurate registration obtained by using the DARTEL algorithm, no DLB-specific pattern of GM atrophy was detected. On the other hand, as 


\begin{tabular}{|c|c|c|c|c|c|}
\hline \multirow[b]{2}{*}{ Comparison and Brain Region } & \multicolumn{4}{|c|}{ Talairach Coordinates } & \multirow[b]{2}{*}{$t$ Value } \\
\hline & Side & $x$ & $\mathrm{Y}$ & Z & \\
\hline \multicolumn{6}{|l|}{ Controls $>$ AD patients } \\
\hline Medial temporal lobe & $\mathrm{Lt}$ & -25 & -27 & -16 & 6.30 \\
\hline Medial temporal lobe & Rt & 30 & -27 & -7 & 5.88 \\
\hline Fornix & Rt & 3 & -7 & 13 & 5.77 \\
\hline Frontal lobe & $\mathrm{Lt}$ & -30 & 25 & 19 & 4.65 \\
\hline Parieto-occipital lobe & $\mathrm{Lt}$ & -21 & -54 & 33 & 4.33 \\
\hline \multicolumn{6}{|l|}{ DLB patients $>$ AD patients } \\
\hline Medial temporal lobe & $\mathrm{Lt}$ & -25 & -20 & -18 & 7.02 \\
\hline Medial temporal lobe & Rt & 27 & -27 & -15 & 6.09 \\
\hline Fornix & $\mathrm{Lt}$ & -1 & -6 & 11 & 4.69 \\
\hline Frontal lobe & Rt & 6 & 23 & 53 & 3.63 \\
\hline Temporal lobe & Rt & 45 & 2 & -22 & 3.45 \\
\hline Medial parieto-occipital lobe & $\mathrm{Rt}$ & 9 & -50 & 22 & 3.28 \\
\hline
\end{tabular}

Table 5: Regions in which conventional VBM identified significantly reduced WM: comparisons between patients and normal controls

\begin{tabular}{llrrrrr}
\hline & \multicolumn{5}{c}{ Talairach Coordinates } & \\
\cline { 2 - 4 } Comparison and Brain Region & Side & X & Y & Z & $t$ Value \\
\hline Controls $>$ DLB patients & & & & & \\
$\quad$ Medial temporal lobe & Rt & 34 & -41 & 2 & 4.98 \\
$\quad$ Medial temporal lobe & Lt & -32 & -31 & -4 & 3.97 \\
Controls $>$ AD patients & & & & & \\
$\quad$ Medial temporal lobe & Rt & 34 & -43 & 2 & 7.71 \\
$\quad$ Parietal lobe & Lt & -16 & -46 & 47 & 4.74 \\
$\quad$ Frontal lobe & Rt & 12 & 35 & 44 & 4.50 \\
DLB patients $>$ AD patients & & & & & \\
$\quad$ Medial temporal lobe & Lt & -24 & -24 & -16 & -6.76 \\
$\quad$ Medial temporal lobe & Rt & 24 & -17 & -21 & 5.82 \\
$\quad$ Parietal lobe & Lt & -48 & -24 & 46 & 3.68 \\
\hline
\end{tabular}
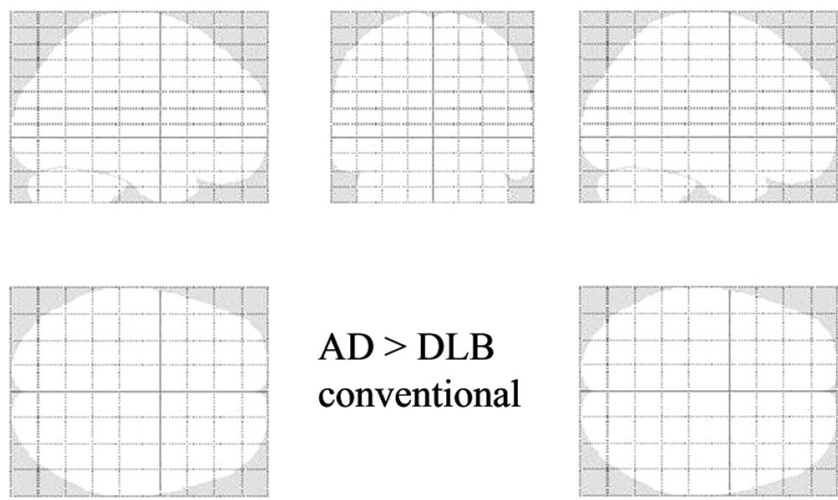

\section{$\mathrm{AD}>\mathrm{DLB}$ conventional}
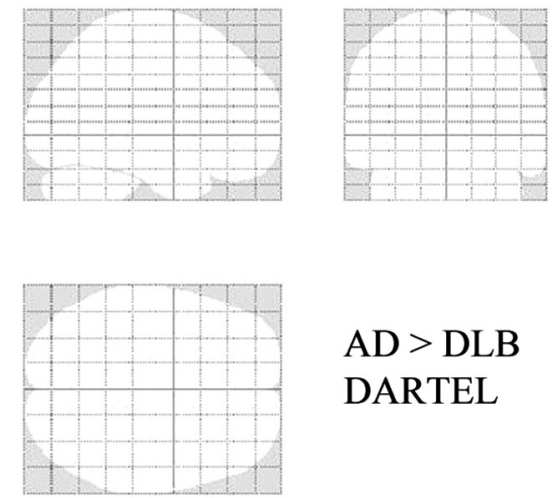

Fig 4. Statistical parametric maps comparing the brains of patients with DLB and patients with AD. Highlighted areas represent regions with significantly decreased $W M$ in patients with $\mathrm{AD}$ compared with those with $\mathrm{DLB}(P<.001$ uncorrected). The MTLs and parieto-occipital deep brain regions are detected in both conventional VBM (lower left) and VBM-DARTEL (lower right). In comparison with patients with $A D$, patients with $D L B$ show no regions with significantly decreased WM $(P<.001$, uncorrected $)$.
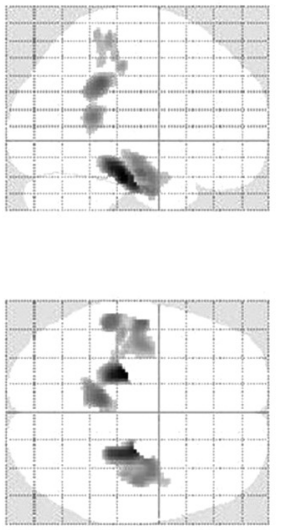

\section{$\mathrm{DLB}>\mathrm{AD}$ conventional}
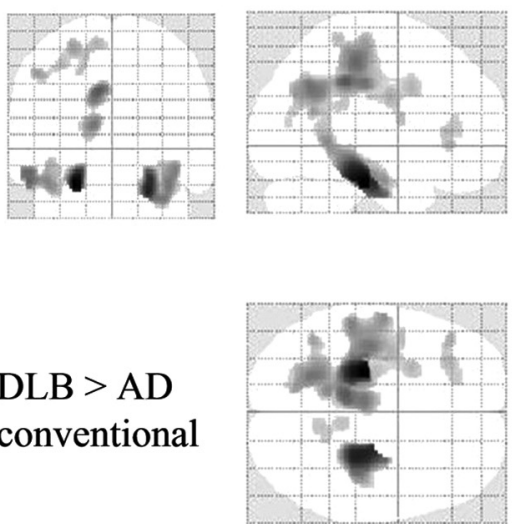

\section{$\mathrm{AD}>\mathrm{DLB}$ \\ DARTEL}

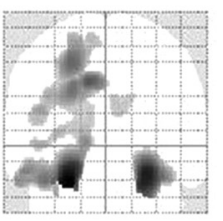

\section{DLB $>$ AD \\ DARTEL}

established in previous studies, greater loss of GM in the MTLs was seen in patients with DLB compared with healthy controls (Fig 1). However, the degree of GM reduction in the temporal region was much smaller in DLB than in AD (Fig 2). These results are in agreement with those of previous region-ofinterest $^{11-13}$ and recent VBM ${ }^{18,19}$ studies. A greater loss of GM in $\mathrm{AD}$ could help explain the results of a neurophysiological study in which there was more severe episodic memory impairment in $\mathrm{AD}$ than in DLB. ${ }^{24,25}$

Although VBM-DARTEL revealed that WM was preserved in patients with DLB (compared with healthy controls), conventional VBM identified bilateral WM atrophy in the MTLs and periventricular regions (Fig 3). The periventricular decrease could be attributed to the improper deformation of
WM segments, which was probably caused by GM atrophy in the MTLs. Previous studies found WM abnormalities mainly by examining diffusion tensor images. ${ }^{26-30}$ However, these results were still not consistent. In the present study, no significant decrease in WM in patients with DLB was found, despite the considerable severity of their condition (Table 1). While both forms of dementia may involve bilateral GM atrophy in the MTLs, preservation of WM may aid in differentiating DLB from $\mathrm{AD}$. Additional comparisons between DLB and AD on other stages should be analyzed in the future.

VBM-DARTEL - based comparisons between healthy controls (Fig 3) and patients with DLB and AD (Fig 4) revealed that patients with $\mathrm{AD}$ show a bilateral decrease in WM volume in the MTLs, parieto-occipital lobes, and frontal lobes; addi- 
tional decreases in the periventricular region and scattered decreases in the parietal lobe were identified with conventional VBM. In addition to the WM decrease in the MTLs, the WM decrease in the periventricular area identified with conventional VBM was similar to that shown in several previous VBM studies. Li et $\mathrm{al}^{31}$ found that patients with AD had decreased WM in the corpus callosum and temporal lobes. Balthazar et $\mathrm{al}^{32}$ found WM atrophy in periventricular areas, the corpus callosum, and regions adjacent to the associative cortices. These studies attributed the WM loss in the corpus callosum to the disconnection of WM fibers. Contrary to previous studies, our VBM-DARTEL analysis did not identify a WM decrease in the periventricular region. The scattered WM decrease found with conventional VBM might have been the result of incomplete registration to the templates. The present study found VBM-DARTEL to be more accurate than conventional VBM in differentiating DLB from AD. However, the only problem in VBM-DARTEL is that it is time-consuming; the whole procedure requires a week for processing the analysis, especially when creating a subject-specific template.

A limitation of the present study is that diagnoses made by using MR imaging data can only be confirmed with postmortem examinations. However, this limitation was overcome by the large sample size, highly matched ages, MMSE scores of subjects from different groups, and clinical confirmation of diagnoses facilitated by following patients for $>3$ years. Nevertheless, future postmortem investigations may help to clarify the accuracy of our results. More important, it should be recognized that the VBM-DARTEL method is still being developed, and further advances in precision and accuracy may enable DLB and AD to be differentiated more finely. Future longitudinal follow-up is warranted.

\section{Conclusions}

Analysis with VBM-DARTEL was more accurate and resulted in the detection of more localized morphologic alterations than analysis with conventional VBM. WM preservation in DLB could help to differentiate this condition from AD. In the future, DARTEL-VBM could prove to be more accurate, especially in longitudinal studies because of the absence of the confounding effect of misregistration.

\section{References}

1. McKeith IG, Dickson DW, Lowe J, et al. Diagnosis and management of dementia with Lewy bodies: third report of the DLB Consortium. Neurology 2005;65: 1863-72. Epub 2005 Oct 19

2. Ishii K, Imamura T, Sasaki M, et al. Regional cerebral glucose metabolism in dementia with Lewy bodies and Alzheimer's disease. Neurology 1998;51: 125-30

3. Higuchi M, Tashiro M, Arai H, et al. Glucose hypometabolism and neuropathological correlates in brains of dementia with Lewy bodies. Exp Neurol 2000;162:247-56

4. Minoshima S, Foster NL, Sima AA, et al. Alzheimer's disease versus dementia with Lewy bodies: cerebral metabolic distinction with autopsy confirmation. Ann Neurol 2001;50:358-65
5. Ishii K, Hosaka K, Mori T, et al. Comparison of FDG-PET and IMP-SPECT in patients with dementia with Lewy bodies. Ann Nucl Med 2004;18:447-51

6. Ishii K, Yamaji S, Kitagaki H, et al. Regional cerebral blood flow difference between dementia with Lewy bodies and AD. Neurology 1999;53:413-16

7. Lobotesis K, Fenwick JD, Phipps A, et al. Occipital hypoperfusion on SPECT in dementia with Lewy bodies but not AD. Neurology 2001;56:643-49

8. Small GW. Neuroimaging as a diagnostic tool in dementia with Lewy bodies. Dement Geriatr Cogn Disord 2004;17(suppl 1):25-31

9. Ishii $\mathrm{K}$, Soma $\mathrm{T}$, Kono $\mathrm{AK}$, et al. Comparison of regional brain volume and glucose metabolism between patients with mild dementia with Lewy bodies and those with mild Alzheimer's disease. J Nucl Med 2007;48:704-11

10. Kono AK, Ishii K, Sofue K, et al. Fully automatic differential diagnosis system for dementia with Lewy bodies and Alzheimer's disease using FDG-PET and 3D-SSP. Eur J Nucl Med Mol Imaging 2007;34:1490-97

11. Hashimoto M, Kitagaki H, Imamura T, et al. Medial temporal and whole-brain atrophy in dementia with Lewy bodies: a volumetric MRI study. Neurology 1998;51:357-62

12. Harvey GT, Hughes J, McKeith IG, et al. Magnetic resonance imaging differences between dementia with Lewy bodies and Alzheimer's disease: a pilot study. Psychol Med 1999;29:181-87

13. Barber R, Gholkar A, Scheltens P, et al. Medial temporal lobe atrophy on MRI in dementia with Lewy bodies. Neurology 1999;52:1153-58

14. Ashburner J, Friston KJ. Voxel-based morphometry: the methods. Neuroimage 2000;11:805-21

15. Good CD, Johnsrude IS, Ashburner J, et al. A voxel-based morphometric study of ageing in 465 normal adult human brains. Neuroimage 2001;14(1 pt 1): 21-36

16. Ashburner J, Friston KJ. Unified segmentation. Neuroimage 2005;26:839-51

17. Burton EJ, Karas G, Paling SM, et al. Patterns of cerebral atrophy in dementia with Lewy bodies using voxel-based morphometry. Neuroimage 2002;17:618-30

18. Burton EJ, McKeith IG, Burn DJ, et al. Cerebral atrophy in Parkinson's disease with and without dementia: a comparison with Alzheimer's disease, dementia with Lewy bodies and controls. Brain 2004;127:791-800

19. Whitwell JL, Weigand SD, Shiung MM, et al. Focal atrophy in dementia with Lewy bodies on MRI: a distinct pattern from Alzheimer's disease. Brain 2007;130:708-19

20. Cousins DA, Burton EJ, Burn D, et al. Atrophy of the putamen in dementia with Lewy bodies but not Alzheimer's disease: an MRI study. Neurology 2003;61:1191-95

21. Brenneis $\mathrm{C}$, Wenning $\mathrm{GK}$, Egger $\mathrm{KE}$, et al. Basal forebrain atrophy is a distinctive pattern in dementia with Lewy bodies. Neuroreport 2004;15:1711-14

22. Ashburner J. A fast diffeomorphic image registration algorithm. Neuroimage 2007;38:95-113. Epub 2007 Jul 18

23. McKeith IG, Galasko D, Kosaka K, et al. Consensus guidelines for the clinical and pathologic diagnosis of dementia with Lewy bodies (DLB): report of the consortium on DLB international workshop. Neurology 1996;47:1113-24

24. Calderon J, Perry RJ, Erzinclioglu SW, et al. Perception, attention, and working memory are disproportionately impaired in dementia with Lewy bodies compared with Alzheimer's disease. J Neurol Neurosurg Psychiatry 2001;70:157-64

25. Ferman TJ, Smith GE, Boeve BF, et al. Neuropsychological differentiation of dementia with Lewy bodies from normal aging and Alzheimer's disease. Clin Neuropsychol 2006;20:623-36

26. Ota M, Sato N, Ogawa M, et al. Degeneration of dementia with Lewy bodies measured by diffusion tensor imaging. NMR Biomed 2009;22:280-84

27. Firbank MJ, Blamire AM, Krishnan MS, et al. Atrophy is associated with posterior cingulate white matter disruption in dementia with Lewy bodies and Alzheimer's disease. Neuroimage 2007;36:1-7

28. Firbank MJ, Blamire AM, Krishnan MS, et al. Diffusion tensor imaging in dementia with Lewy bodies and Alzheimer's disease. Psychiatry Res 2007;155:135-45

29. Bozzali M, Falini A, Cercignani M, et al. Brain tissue damage in dementia with Lewy bodies: an in vivo diffusion tensor MRI study. Brain 2005;128:1595-604

30. Burn DJ, O'Brien JT. Use of functional imaging in Parkinsonism and dementia. Mov Disord 2003;18(suppl 6):S88-95

31. Li S, Pu F, Shi F, et al. Regional white matter decreases in Alzheimer's disease using optimized voxel-based morphometry. Acta Radiol 2008;49:84-90

32. Balthazar ML, Yasuda CL, Pereira FR, et al. Differences in grey and white matter atrophy in amnestic mild cognitive impairment and mild Alzheimer's disease. Eur J Neurol 2009;16:468-74 